\title{
Temperature dependence of the double layer capacitance for the restricted primitive model of an electrolyte solution from a density functional approach
}

\author{
Douglas Henderson \\ J. Reszko-Zygmunt \\ Stefan Sokolowski \\ Dezso Boda
}

Follow this and additional works at: https://scholarsarchive.byu.edu/facpub

Part of the Biochemistry Commons, and the Chemistry Commons

\section{Original Publication Citation}

Zygmunt, Reszko J., Soko S. Owski, D. Henderson, and D. Boda."Temperature dependence of the double layer capacitance for the restricted primitive model of an electrolyte solution from a density functional approach." The Journal of Chemical Physics 122 (2

\section{BYU ScholarsArchive Citation}

Henderson, Douglas; Reszko-Zygmunt, J.; Sokolowski, Stefan; and Boda, Dezso, "Temperature dependence of the double layer capacitance for the restricted primitive model of an electrolyte solution from a density functional approach" (2005). Faculty Publications. 393.

https://scholarsarchive.byu.edu/facpub/393

This Peer-Reviewed Article is brought to you for free and open access by BYU ScholarsArchive. It has been accepted for inclusion in Faculty Publications by an authorized administrator of BYU ScholarsArchive. For more information, please contact ellen_amatangelo@byu.edu. 


\title{
Temperature dependence of the double layer capacitance for the restricted primitive model of an electrolyte solution from a density functional approach
}

\author{
J. Reszko-Zygmunt ${ }^{\mathrm{a})}$ and S. Sokołowski ${ }^{\mathrm{b})}$ \\ Department for the Modelling of Physico-Chemical Processes, Faculty of Chemistry UMCS, 20031 Lublin, \\ Poland \\ D. Henderson ${ }^{\mathrm{c})}$ \\ Department of Chemistry and Biochemistry, Brigham Young University, Utah 84602-5700 \\ D. Boda ${ }^{\text {d) }}$ \\ Department of Physical Chemistry, University of Veszprém, P.O. Box 158, H-8201 Veszprém, Hungary
}

(Received 14 October 2004; accepted 29 November 2004; published online 15 February 2005)

\begin{abstract}
We apply a different version of the density functional theory, given by Pizio, Patrykiejew, and Sokołowski [J. Chem. Phys. 121, 11957 (2004)], for a nonuniform restricted primitive model of an electrolyte solution to evaluate the temperature dependence of the capacitance of an electric double layer. We show that this theory is capable of reproducing the computer simulation data at a quantitative level. In particular, the reversal of the temperature dependence of the capacitance at low temperatures is predicted. This phenomenon has been difficult to predict from theory. Further, this theory also leads to an accurate description of the double layer structure. (C) 2005 American Institute of Physics. [DOI: 10.1063/1.1850453]
\end{abstract}

\section{INTRODUCTION}

One of the puzzling problems in the theory of an electrical double layer is connected with an explanation of the changes of the double layer electrical capacitance near the point of zero charge with the temperature for different interfaces. Early investigations ${ }^{1-3}$ drew attention to the opposite temperature dependence of the capacitance for aqueous electrolytes and for molten salts. The capacitance was found to increase with increasing temperature for molten salts, whereas for aqueous solutions an inverse effect was usually observed. Since molten salts have significantly higher ionic density, this behavior was interpreted as a density effect. However, computer simulations of the so-called restricted primitive model (RPM) of an electrolyte near a charged hard wall carried out by Boda and co-workers ${ }^{4-7}$ for a wide temperature and density range showed that the capacitance of the interface is quantitatively similar in the high and low density regimes. In both cases, the capacitance has a negative slope at high temperatures and a positive slope at low temperatures. Therefore, the results of Boda et al. imply that the behavior of the capacitance at low temperatures is not a density but a temperature effect.

There have been attempts to explain and reproduce the results of the simulations of Boda and co-workers ${ }^{4-7}$ using the application of different theoretical approaches. ${ }^{7-9}$ Unfortunately, the usual density functional (DF) approaches, ${ }^{9-15}$ as well as the singlet mean spherical approximation (MSA)

\footnotetext{
${ }^{a)}$ Electronic mail: jreszko@hermes.umcs.lublin.pl

b)Electronic mail: stefan@zool.umcs.lublin.pl

${ }^{c}$ Electronic mail: doug@chem.byu.edu

d)Electronic mail: boda@almos.vein.hu
}

(Refs. 5 and 7) and generalized MSA (GMSA) (Ref. 8) have failed to reproduce simulation data. However, the employment ${ }^{7}$ of the concept of ionic association in a MSAtype approach was partially successful in explaining the anomalous temperature dependence of the capacitance of the electrical double layer of electrolyte solutions. In particular, it was demonstrated that, except in the intermediate region of temperatures where the temperature derivative of the capacitance changes sign, a Bjerrum-like ${ }^{16}$ correction of the MSA with Ebeling's ${ }^{17}$ expression for the associative constant, ${ }^{18}$ satisfactory reproduces the computer simulation data at high and at low temperatures. The last finding suggests that the DF theories cited above do not properly account for the contribution arising from electrostatic interactions in the system. More precisely, there is "too little attraction" in the system and an associative free energy term would correct problem.

Any DF theory determines thermodynamic properties of an inhomogeneous fluid from the Helmholtz free energy $F$ and its functional dependence on the local densities of particles $\left\{\rho_{i}(\mathbf{r})\right\}$. The free energy functional is commonly decomposed into the sum of the ideal $F_{\text {id }}$, the hard-sphere $F_{\text {hs }}$, and the electrostatic $F_{\text {el }}$ contributions. Various formulations of the DF theory have been discussed in the literature. They differ by the expressions for $F_{\mathrm{hs}}$ and $F_{\mathrm{el}}$. To our knowledge, the majority of the DF theories ${ }^{9-15}$ have followed a perturbational formulation of the electrostatic free energy functional with respect to a bulk homogeneous fluid and used the expression resulting from the MSA bulk theory to describe the direct two-particle correlation function. In general, one can treat these theories as following in this respect the MSA "compressibility route." It is thus not surprising that despite of a great number of publications in which DF theories have 
been applied in studies of several aspects of adsorption of ions, all of the above cited works fail to describe surfaceinduced phase transitions. However, it should be noted here that the problem of the description of the RPM systems containing liquidlike and gaslike phases, as well as an interface between them has been considered by Groh, Evans, and Dietrich, ${ }^{19}$ Telo da Gama, Evans, and Sluckin, ${ }^{20}$ and by Weiss and Schroer. ${ }^{21}$ The DF approximation of Groh, Evans, and Dietrich ${ }^{19}$ was based on the incorporation of the contribution due to electrostatic interactions into the grand potential functional using the pair correlation functions of the homogeneous fluid, as obtained from the bulk MSA theory. In other words, the approach of Groh, Evans, and Dietrich ${ }^{19}$ followed the MSA "energy route" ${ }^{, 22,23}$ and thus was able to describe the liquid-vapor phase transition in ionic systems. The two remaining approaches ${ }^{20,21}$ used the square-gradient approximation for the density functional. Basically, square gradient type theories should be used to describe the systems where the density gradients are not too large. This is not the case in the electrical double layer.

Recently Pizio, Patrykiejew, and Sokołowski ${ }^{24}$ proposed a new version of the DF theory, which is able to describe phase transitions in RPM fluids confined in slitlike pores. They applied an analytical expression resulting from the energy route of the bulk MSA theory ${ }^{18,22,23}$ for the electrostatic part of the free energy. The electrostatic functional was written down similarly to the hard-sphere contribution (or, e.g., to the associative free energy contribution in the case of uncharged but associating fluids, see, e.g., Ref. 25). This was possible by introducing suitably chosen "electrostatic" averaged densities. In this note we apply this approach to reproduce simulation data of Boda et al. ${ }^{4-6}$ for electric double layer of electrolyte solutions.

\section{THEORY}

We recall that we restrict our attention to a binary mixture of ionic species. Using the ion diameter to scale distances, the symbols $d_{i}=d=1, Z_{i}$ and $\mu_{i}$ denote, respectively, the hard-sphere diameter, valence of ions, and the chemical potential of species $(i=1,2)$. Moreover, in this work we consider 1:1 electrolyte, $Z_{1}=-Z_{2}=1$. The fluid in contact with a hard charged wall and the wall surface charge density is $\sigma$. The interaction between the ions is

$$
u_{i j}(r)=\left\{\begin{array}{l}
\infty, \quad r<1 \\
e^{2} Z_{i} Z_{j} / \varepsilon r, \quad r>1,
\end{array}\right.
$$

where $r$ is the distance between a pair of ions. We also assume that the dielectric constant $\epsilon$ is uniform throughout the entire system. The interaction between the ions of species $i$ and the wall is given by

$$
u_{i}(z)=v_{i}(z)+w_{i}(z),
$$

where $v_{i}(z)$ and $w_{i}(z)$ are, respectively, the nonelectrostatic and the electrostatic contributions to the external potential,

$$
v_{i}(z)= \begin{cases}0, & z>d / 2 \\ \infty, & \text { otherwise }\end{cases}
$$

and

$$
w_{i}(z)=-\left(4 \pi \sigma Z_{i} e / \varepsilon\right) z .
$$

The electrostatic potential $\Psi(z)$ is determined by Poisson's equation,

$$
\nabla^{2} \Psi(z)=-\frac{4 \pi e}{\varepsilon} \sum_{i=1,2} Z_{i} \rho_{i}(z),
$$

where $\rho_{i}(z)$ are the density profiles of ionic species. The integration of Poisson's equation is carried out assuming that the electrostatic potential at the wall is $V=\Psi(z=0)$. The relation between the density profiles and the surface charge density is given by

$$
\sigma / e=-\sum_{i=1,2} Z_{i} \int \rho_{i}(z) d z .
$$

The above described system is studied using DF theory. The grand potential of an inhomogeneous fluid is written in the form ${ }^{26,27}$

$$
\begin{aligned}
\Omega= & F\left[\left\{\rho_{i}\right\}\right]+\frac{1}{2} \sum_{i=1,2} e Z_{i} \int \rho_{i}(z) \Psi(z) d \mathbf{r} \\
& +\sum_{i=1,2} \int \rho_{i}(z)\left[u_{i}(z)-\mu_{i}\right] d \mathbf{r},
\end{aligned}
$$

where the free energy functional $F\left[\left\{\rho_{i}\right\}\right]$ is decomposed into ideal (id), hard sphere (hs), and electrostatic (el) terms as follows:

$$
F\left[\left\{\rho_{i}\right\}\right]=F_{\mathrm{id}}\left[\left\{\rho_{i}\right\}\right]+F_{\mathrm{hs}}\left[\left\{\rho_{i}\right\}\right]+F_{\mathrm{el}}\left[\left\{\rho_{i}\right\}\right] .
$$

The ideal term is known exactly, $F_{\text {id }}\left[\left\{\rho_{i}\right\}\right]$ $=\Sigma_{i=1,2}\left[\rho_{i}(z) \ln \rho_{i}(z)-\rho_{i}(z)\right]$. For the hard-sphere term, however, we apply the expression resulting from a recent version $^{28}$ of the fundamental measure theory, ${ }^{29}$

$$
F_{\mathrm{hs}}\left[\left\{\rho_{i}\right\}\right]=\int d \mathbf{r} F_{\mathrm{hs}}\left[\left\{n_{\alpha}\right\}\right],
$$

with the free energy density consisting of terms dependent on scalar and vector weighted densities, $n_{\alpha}(\mathbf{r}) \quad(\alpha$ $=0,1,2,3)$ and $\mathbf{n}_{\alpha}(\mathbf{r})(\alpha=V 1, V 2),{ }^{26-29}$

$$
\begin{aligned}
f_{\text {(hs) }}= & -n_{0} \ln \left(1-n_{3}\right)+\frac{n_{1} n_{2}-\mathbf{n}_{V 1} \cdot \mathbf{n}_{V 2}}{1-n_{3}} \\
& +n_{2}^{3}\left(1-3 \xi^{2}\right) \frac{n_{3}+\left(1-n_{3}\right)^{2} \ln \left(1-n_{3}\right)}{36 \pi n_{3}^{2}\left(1-n_{3}\right)^{2}},
\end{aligned}
$$

where $\xi(\mathbf{r})=\left|\mathbf{n}_{V 2}(\mathbf{r})\right| / n_{2}(\mathbf{r})$. In Eqs. (10), (12), and (13) the spatial dependence of all variables has been suppressed, for the sake of simplicity. We have neglected the tensor contribution to the free energy. ${ }^{30}$ Any possible inaccuracy introduced by this approximation is negligible for the problem in question.

The weighted densities, $n_{\alpha}(\mathbf{r}), \alpha=0,1,2,3, V 1, V 2$, are defined as

$$
n_{\alpha}(\mathbf{r})=\sum_{i=1,2} n_{\alpha i}(\mathbf{r})=\sum_{i=1,2} \int d \mathbf{r}^{\prime} \rho_{i}\left(\mathbf{r}^{\prime}\right) w_{\alpha i}\left(\mathbf{r}-\mathbf{r}^{\prime}\right),
$$

where $w_{\alpha i}(\mathbf{r})$ are given in Ref. 26-29. 
In the previously used DF theories approaches, ${ }^{9,11-15}$ the electrostatic contribution to the free energy $F_{\text {el }}\left[\left\{\rho_{i}\right\}\right]$ has had the form of integrals over the short-range part of the direct correlation functions of ionic species. However, in this work we follow the approach of Pizio, Patrykiejew, and Sokołowski $^{24}$ and use the following representation of this functional:

$$
F_{\mathrm{el}}\left[\left\{\boldsymbol{\rho}_{i}\right\}\right]=\int d \mathbf{r} f_{\mathrm{el}}\left[\left\{\overline{\boldsymbol{\rho}_{i}}(z)\right\}\right],
$$

where $\overline{\rho_{i}}(z)$ denote suitably defined inhomogeneous average densities of a reference fluid. One of the simplest possible choices of the form for $f_{\mathrm{el}}\left[\left\{\overline{\rho_{i}}(z)\right\}\right]$ follows from the application of the expression resulting from the MSA equation of state evaluated via the energy route, due originally to Waisman and Lebowitz and restated recently, ${ }^{18}$

$$
f_{\mathrm{el}}\left[\left\{\rho_{i}\right\}\right] / k T=-\frac{e^{2}}{\epsilon k T}\left[Z_{1}^{2} \rho_{1}+Z_{2}^{2} \rho_{2}\right] \frac{\Gamma}{1+\Gamma}+\frac{\Gamma^{3}}{3 \pi},
$$

where

$$
\Gamma=(\sqrt{1+2 \kappa d}-1) / 2 d
$$

and where $\kappa$ denotes the inverse Debye screening length,

$$
\kappa^{2}=\left(4 \pi e^{2} / \epsilon k T\right)\left[Z_{1}^{2} \rho_{1}+Z_{2}^{2} \rho_{2}\right] .
$$

The last three expressions written above correspond to an electroneutral fluid. Therefore, the reference fluid should be defined in such a way that the averaged densities $\left\{\overline{\rho_{i}}(z)\right\}$ must not violate the electroneutrality condition at any point $\mathbf{r}$, because otherwise Eq. (13) could not be applied. The problem of selection of the reference system can be solved ${ }^{24}$ following the development of Gillespie et al.. ${ }^{26,27}$ Thus, we define the weighted densities $\left\{\overline{\rho_{i}}(z)\right\}$ by

$$
\tilde{\rho}_{i}(z)=\int \rho_{i}(z) W_{i}\left(\left|\mathbf{r}=\mathbf{r}^{\prime}\right|\right) d \mathbf{r}^{\prime},
$$

where $W_{i}(r)$ is a weight function,

$$
W_{i}\left(\left|\mathbf{r}-\mathbf{r}^{\prime}\right|\right)=\frac{\theta\left[\left|\mathbf{r}-\mathbf{r}^{\prime}\right|-R_{f}\left(\mathbf{r}^{\prime}\right)\right]}{(4 \pi / 3) R_{f}^{3}\left(\mathbf{r}^{\prime}\right)} .
$$

The radius of the sphere over which the averaging is performed $R_{f}$ is not a well-defined quantity and it is approximated by the "capacitance" radius, i.e., by the ion radius plus the screening length

$$
R_{f}(\mathbf{r})=\frac{d}{2}+\frac{1}{2 \Gamma\left(\left\{\tilde{\rho}_{i}(\mathbf{r})\right\}\right)} .
$$

Imposing the additional requirement ${ }^{26,27}$ that the reference fluid has the same ionic strength as the system with weighted densities $\tilde{\rho}_{i}(z)$, we find that in the case of symmetric 1-1 electrolyte the averaged densities $\left\{\overline{\rho_{i}}(z)\right\}$ are given as

$$
\overline{\rho_{1}}(z)=\overline{\rho_{2}}(z)=\frac{\left[\overline{\rho_{1}}(z)+\overline{\rho_{2}}(z)\right]}{2} .
$$

A general definition of the averaged densities $\left\{\overline{\rho_{i}}(z)\right\}$ for a multicomponent system involving ions of arbitrary valences can be found in Refs. 26 and 27. Because Eqs. (17)-(19) are coupled, the evaluation of $R_{f}$ requires an iteration procedure. This iteration loop should be carried out in addition to the main iteration procedure for evaluating the density profiles, as described in details in Refs. 26 and 27. It is worth noting that the idea of using different weighted densities to calculate the hard-sphere and the electrostatic contributions to the free energy functional has been used by Patra and Ghosh. ${ }^{13,14}$ The latter approach has followed the Denton-Ashcroft formulation $^{31}$ of DF theory for simple fluids.

Far from the surface the fluid density is constant and equal the bulk fluid density, $\rho_{b}=\rho_{b, 1}+\rho_{b, 2}$. The grand thermodynamic potential of the bulk RPM, $\Omega_{b}$, is calculated according to Eqs. (7)-(13), in which the density profiles are substituted by constant bulk densities, $\left\{\rho_{b, i}\right\}$. In the calculation of the properties of the bulk system, the electrostatic potential $\Phi$ is set equal to zero.

The density profiles are obtained by minimizing the excess grand potential functional $\Delta \Omega=\Omega-\Omega_{b}$. Because the resulting equations have already been presented by Pizio, $\mathrm{Pa}-$ trykiejew, and Sokołowski, ${ }^{24}$ we can omit them here. The local densities were found employing an iterational procedure and all the integration were carried out using Simpson method with the grid size of $0.02 d$. For the sake of convenience the electric potential on the pore walls $V$ has been chosen as a variable that specifies the system, as common in DF calculations. Consequently, the charge density on the pore walls $\sigma$ results from an integration of the calculated profiles.

\section{RESULTS AND DISCUSSION}

The capacitance $C$ and the differential capacitance $C_{D}$ are defined as follows: ${ }^{7}$

$$
C=\frac{\sigma}{V}, \quad C_{D}=\frac{\partial \sigma}{\partial V} .
$$

However, near the point of zero charge, where the potential $V$ is a linear function of the charge density of the electrode $\sigma$, these two capacitances become equal. In this work we will confine ourselves to small $\sigma$ and will not distinguish between $C$ and $C_{D}$ and will use only the term capacitance.

Before discussing the dependence of $C$ on the temperature, first we will show an example of the predictions of the double layer structure. For this purpose we have selected a case where the previous version of the density functional approach $^{9}$ failed in reproducing the density profiles.

In Fig. 1(a) we compare the local densities of ions in contact with a single charged wall. The reduced surface charge density is $\sigma^{*}=\sigma d^{2} / e=0.00765$ and the bulk density and the reduced temperature are $\rho_{b}^{*}=\rho_{b} d^{3}=0.04$ and $T^{*}$ $=k T \epsilon d / e^{2}=0.15$, respectively. It is evident that the present theory provides much better agreement with simulations than the theory outlined in Ref. 9. In particular, in contrast to the previous approach, the profiles of both ionic species exhibit a depletion near the wall, in agreement with the simulations. This is a quantitative change in comparison with previous predictions. Further insight can be gained from the contact values of the profiles at zero electrode charge that are plotted in Fig. 1(b) for $\rho_{b} d^{3}=0.04$. The agreement with simulations 

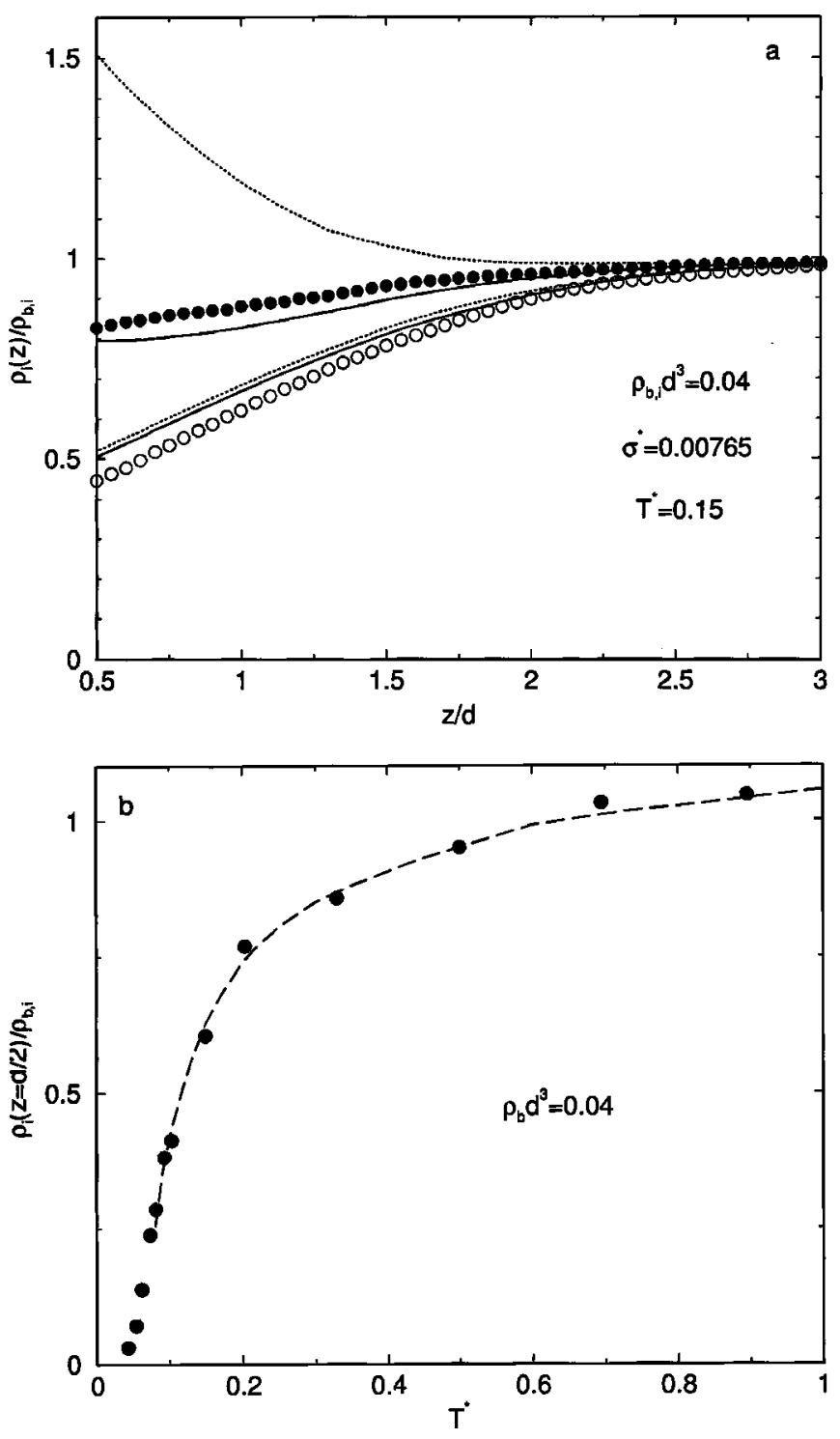

FIG. 1. (a) A comparison of the counterion and coion density profiles at a charged wall from the present theory (solid lines), from the DF theory of Boda et al. (Ref. 9) (dotted lines) and from Monte Carlo simulations (filled and empty circles) (Ref. 6). (b) A comparison of our theoretical predictions of the local density contact values (dashed curve) with the simulation data of Henderson, Boda, and Wasan (Ref. 8) (circles) carried out for an uncharged wall. All the parameters are given in the figure.

is good. Note that the DF calculations were not made for temperatures below $T^{*}=0.08$. The reason for this is explained below.

Figure 2 presents the temperature dependence of the capacitance at four densities. The points are the results of the computer simulations of Boda et al. ${ }^{6}$ and the curves are the results obtained from different theoretical approaches

The behavior of capacitance at high temperatures is often interpreted within the framework of the Gouy-Chapman theory, which after linearization leads to the expression ${ }^{6}$

$$
C=\frac{\epsilon}{4 \pi} \kappa,
$$

whereas MSA theory ${ }^{6}$ gives

$$
C=\frac{\epsilon \Gamma}{2 \pi}
$$

Neither of the above equations predicts the change of the sign of the temperature derivative of $C$. The GMSA also suffers from this same drawback. ${ }^{8}$

To explain the results of simulations, Holovko et al. ${ }^{7}$ took association into account. There are at least two possible routes for implementing this concept. According to the first approach, a theory (e.g., the MSA theory) is modified correcting the ion density by taking into account only the concentration of free ions and neglecting the contribution from ion pairs. The concentration of free ions is obtained from the mass action law (MAL). ${ }^{32}$ This approach is called the MSA\&MAL theory. Another way ${ }^{33}$ starts from the so-called associative MSA (AMSA). However, the capacitance calculated from AMSA still has a negative slope, even at low temperatures and therefore fails to reproduce the simulations data. In contrast, the MSA\&MAL theory leads to a quantitatively correct prediction of the dependence of the capacitance on the temperature. ${ }^{7}$

Except for the region of quite low temperatures, the DF theory presented reproduces the simulation data quite well. However, it was impossible to carry out the DF calculations at low temperatures. The reason is the following. At temperatures lower than $T_{c}^{*} \approx 0.0786$ (which is the bulk critical temperature for the system in question as given by the MSA energy equation of state of the bulk counterpart of the present theory) a first-order phase transition is predicted. Decreasing the temperature at a fixed density we cross the liquid-vapor coexistence envelope. Consequently, the bulk density $\rho_{b}^{*}=0.019$ [from Fig. 2(a)] lies inside the two-phase region at temperatures lower than $\approx 0.078$. Note that the bulk critical temperature predicted by the MSA is much higher than the critical temperature estimated from simulations, $T_{c}^{*}$ $\approx 0.0496$ (cf. Table I from Ref. 18).

In Fig. 3 we plot the capacitance obtained for different surface charge densities $\sigma$ for the fluid of bulk density $\rho_{b}^{*}$ $=0.040$. For the reasons explained above we have plotted the DF results only at the two highest temperatures. The predictions of the theory are quantitatively similar to the results of simulations. The capacitance decreases linearly as the electrode charge density decreases at a given temperature. Moreover, the slope of the curve at $T^{*}=0.1$ is lower than at $T$ $=0.08$, similarly to simulations.

\section{SUMMARY}

In this work we have demonstrated that the version of the DF approach considered here predicts the temperature dependence of the capacitance of electric double layer of an electrolyte successfully. Moreover, it also reproduces the double layer structure reasonably well. Further development of the theory is also possible. The effort should concentrate on the improvement of the predictions of the critical properties of the bulk RPM fluid by the bulk counterpart of the theory. This means that the next step should rely on the incorporation of the effects due to ion association into the theory. In particular, it is possible to include the associative 

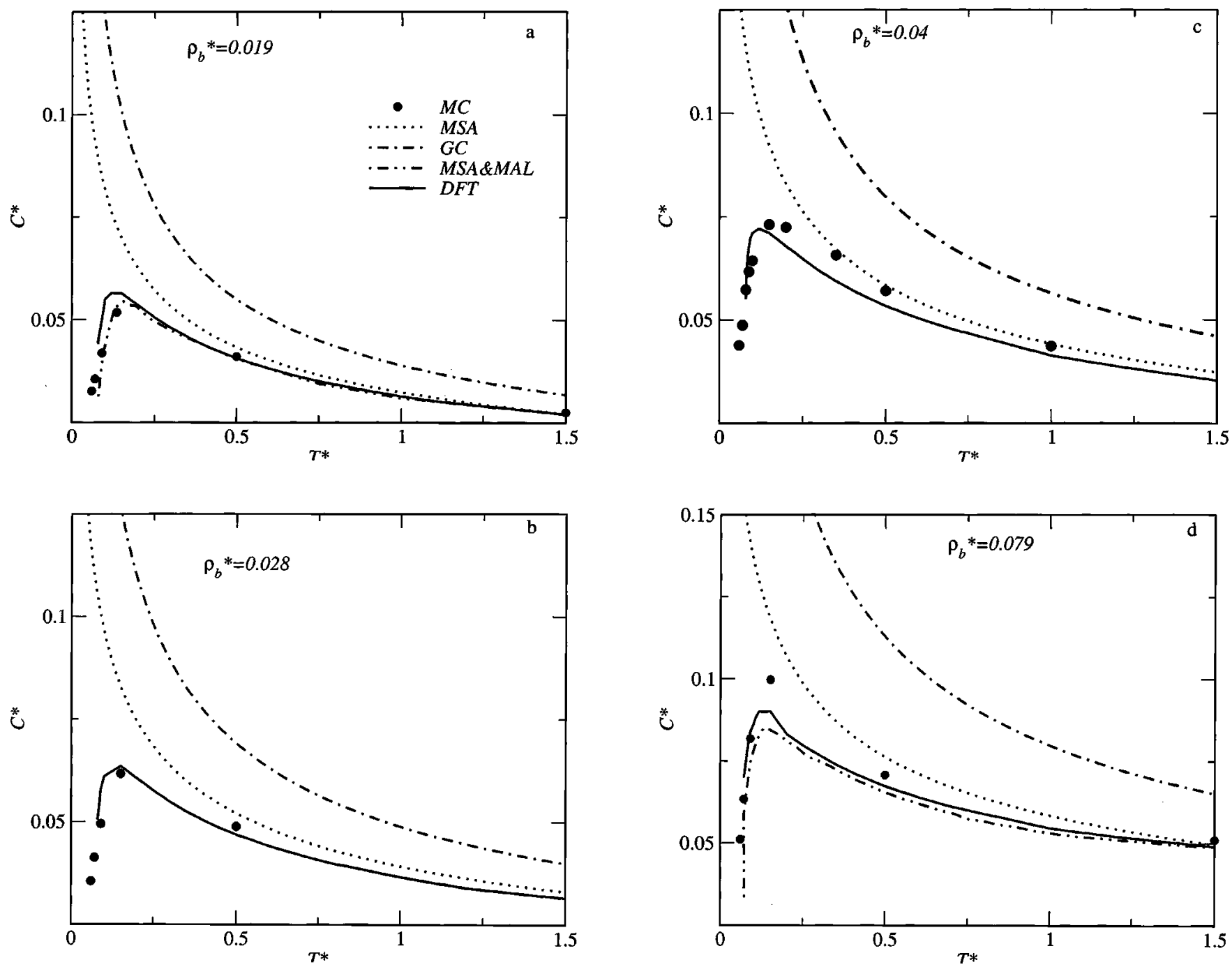

FIG. 2. The reduced capacitance, $C^{*}=C d / \epsilon$, as a function of reduced temperature $T^{*}$ for four reduced densities given in the consecutive parts (a)-(d). Points denote results of Monte Carlo simulations (Ref. 6), the meaning of the lines in explained in (a). Note that MSA \& MAL results are plotted only in (a) and (d).

energy term in the free energy functional ${ }^{34}$ generalizing the approach outlined by Jiang et al. ${ }^{18}$ to the case of nonuniform systems.

Comparisons of the proposed version of the DF theory for other applications can be found in Ref. 24. In all the calculations carried out so far, satisfactory agreement with simulation data has been found.

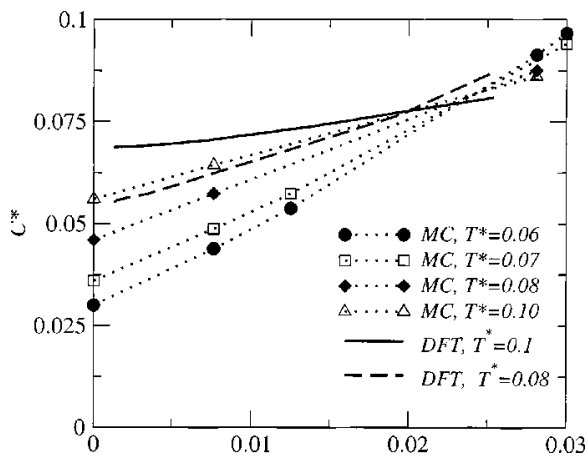

FIG. 3. The reduced capacitance, $C^{*}=C d / \epsilon$, as a function of the reduced surface charge density $\sigma^{*}=\sigma d^{2} / e$. The meaning of all lines is explained in the figure. The reduced density is $\rho^{*}=0.04$.

\section{ACKNOWLEDGMENTS}

S.S. acknowledges EU for funding this work as a TOK Contract No. 509249. D.B. and D.H. acknowledge the support of NATO, Grant No. PST CLG 980366.

${ }^{1}$ K. R. Painter, P. Ballone, M. P. Tosi, P. J. Grout, and N. H. March, Surf. Sci. 133, 89 (1983).

${ }^{2}$ P. Ballone, G. Pastore, M. P. Tosi, K. R. Painter, P. J. Grout, and N. H. March, Phys. Chem. Liq. 13, 269 (1984).

${ }^{3}$ N. H. March and M. P. Tosi, Coulomb Liquids (Academic, London, 1984). ${ }^{4}$ D. Boda, K. Y. Chan, and D. Henderson, J. Chem. Phys. 109, 7362 (1998).

${ }^{5}$ D. Boda, D. Henderson, and K. Y. Chan, J. Chem. Phys. 110, 5346 (1999).

${ }^{6}$ D. Boda, D. Henderson, K. Y. Chan, and D. T. Wasan, Chem. Phys. Lett. 308, 473 (1999).

${ }^{7}$ M. Holovko, V. Kapko, D. Henderson, and D. Boda, Chem. Phys. Lett. 341, 363 (2001).

${ }^{8}$ D. Henderson, D. Boda, and D. T. Wasan, Chem. Phys. Lett. 325, 655 (2000).

${ }^{9}$ D. Boda, D. Henderson, L. Mier y Teran, and S. Sokołowski, J. Phys.: Condens. Matter 14, 11945 (2002)

${ }^{10}$ L. Mier y Teran, D. Boda, D. Henderson, and S. Quimones-Cisneros, Mol. Phys. 99, 1323 (2001)

${ }^{11}$ L. Mier y Teran, S. H. Suh, H. S. White, and H. T. Davis, J. Chem. Phys. 92, 5087 (1990). 
${ }^{12}$ E. Kierlik and M. L. Rosinberg, Phys. Rev. A 42, 3382 (1990).

${ }^{13}$ C. N. Patra and S. K. Ghosh, Phys. Rev. E 47, 4088 (1993).

${ }^{14}$ C. N. Patra, J. Chem. Phys. 111, 9832 (1999).

${ }^{15}$ D. Boda, D. Henderson, R. Rowley, and S. Sokołowski, J. Chem. Phys. 111, 9382 (1999).

${ }^{16}$ N. Bjerrum, Mat. Fys. Medd. K. Dan. Vidensk. Selsk. 7, 1 (1926).

${ }^{17}$ W. Ebeling and M. Grigo, Ann. Phys. (Leipzig) 37, 21 (1980).

${ }^{18}$ J. Jiang, L. Blum, O. Bernard, J. M. Prausnitz, and S. I. Sandler, J. Chem. Phys. 116, 7977 (2002).

${ }^{19}$ B. Groh, R. Evans, and S. Dietrich, Phys. Rev. E 57, 6944 (1998).

${ }^{20}$ M. M. Telo da Gama, R. Evans, and T. J. Sluckin, Mol. Phys. 41, 1355 (1980).

${ }^{21}$ V. C. Weiss and W. Schroer, J. Phys.: Condens. Matter 12, 2637 (2000).

${ }^{22}$ E. Waisman and J. L. Lebowitz, J. Chem. Phys. 52, 4307 (1970).

${ }^{23}$ E. Waisman and J. L. Lebowitz, J. Chem. Phys. 56, 3093 (1972).

${ }^{24}$ O. Pizio, A. Patrykiejew, and S. Sokołowski, J. Chem. Phys. 121, 11957 (2004).
${ }^{25}$ M. Borowko, O. Pizio, and S. Sokołowski, in Computational Methods in Surface and Colloid Science, edited by M. Borowko (Marcel Dekker, New York, 2000), Chap. 4.

${ }^{26}$ D. Gillespie, W. Nonner, and R. S. Eisenberg, J. Phys.: Condens. Matter 14, 12129 (2002).

${ }^{27}$ D. Gillespie, W. Nonner, and R. S. Eisenberg, Phys. Rev. E 68, 031503 (2003).

${ }^{28}$ Y.-X. Yu and J. Z. Wu, J. Chem. Phys. 117, 10156 (2002).

${ }^{29}$ Ya. Rosenfeld, Phys. Rev. Lett. 63, 980 (1989).

${ }^{30}$ A. Cuesta, Y. Martínez-Raton, and P. Tarazona, J. Phys.: Condens. Matter 14, 11965 (2002).

${ }^{31}$ A. R. Denton and N. W. Ashcroft, Phys. Rev. A 39, 426 (1989).

${ }^{32}$ J. Barthel, H. Krienke, W. Kunz, Physical Chemistry of Electrolyte Solutions-Modern Aspects (Steinkopff, Darmstadt, 1998).

${ }^{33}$ H. Krienke, J. Barthel, M. Holovko, I. Protsykevich, and Yu. Kalyuzhnyi, J. Mol. Liq. 87, 191 (2000)

${ }^{34}$ J. Reszko-Zygmunt and S. Sokołowski, J. Electroanal. Chem. (in press). 